PoS $\quad \begin{aligned} & \text { PROCEEDINGS } \\ & \text { OF SCIENCE }\end{aligned}$

\title{
Transport of Solar Energetic Particles across the Parker field due to field line meandering
}

\section{T. Laitinen*}

Jeremiah Horrocks Institute, University of Central Lancashire, UK

E-mail: tlmlaitinen@uclan.ac.uk

\section{A. Kopp}

Theoretische Physik IV, Ruhr-Universität Bochum, Germany

E-mail: aketp4.rub.de

\section{F. Effenberger}

Department of Physics and KIPAC, Stanford University, Stanford, CA, USA

E-mail: feffenestanford.edu

\section{S. Dalla}

Jeremiah Horrocks Institute, University of Central Lancashire, UK

E-mail: sdalla@uclan.ac.uk

\section{M.S. Marsh}

Jeremiah Horrocks Institute, University of Central Lancashire, $U K^{\dagger}$

E-mail: mike.s.marshegmail.com 


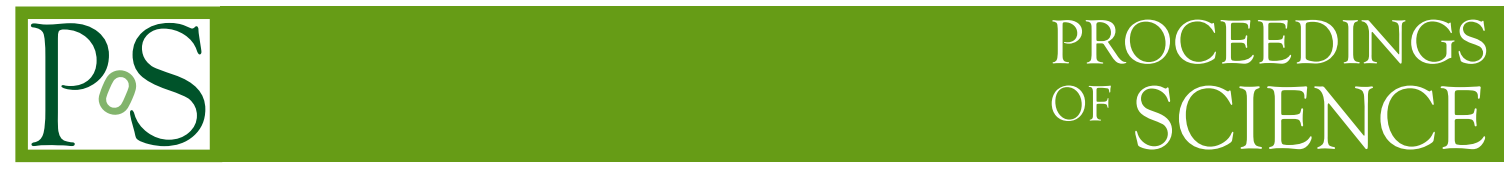

Multi-spacecraft observations of Solar Energetic Particles (SEPs) show that SEPs related to a single solar eruption can be observed over a wide range of heliolongitudes. The SEP anisotropy observations suggest that interplanetary transport significantly contributes to this spreading of SEPs across the mean Parker Spiral field. However, the current transport models that describe the cross-field propagation as diffusion using the Fokker-Planck (FP) equation with a simple crossfield diffusion term, cannot reproduce the extent of SEP events without unrealistically large crossfield diffusion coefficients. Laitinen et al (2013) noted that the initial, non-diffusive propagation of charged particles along turbulently meandering field-lines provides a key to explaining the wide SEP events. Particles that initially propagate along meandering field-lines spread fast across the mean field. Thus, the resulting SEP event extent can be expected to be wider than predicted by the FP description. In this work, we implement field-line meandering into a FP modelling framework for the Parker Spiral geometry. We use an SDE approach to propagate particles along field-lines that meander across the Parker Spiral field, and compare our new model with the traditional FP approach. The particle and field-line diffusion coefficients are calculated using a turbulence model that is consistent with a parallel mean free path of $0.3 \mathrm{AU}$ for $10 \mathrm{MeV}$ protons at $1 \mathrm{AU}$. We find that our new model results in a wide longitudinal extent of SEP events, with $\sigma=33^{\circ}$ for the longitudinal peak intensity distribution, consistent with SEP observations, while for the same turbulence parameters the traditional modelling only gives $\sigma=10^{\circ}$. Our results show that fieldline meandering must be taken into account when modelling SEP propagation in interplanetary space. We discuss the effect of turbulence strength on the extent of SEP events.

The 34th International Cosmic Ray Conference,

30 July- 6 August, 2015

The Hague, The Netherlands

*Speaker.

${ }^{\dagger}$ Now at Met Office, Exeter, UK 


\section{Introduction}

Solar Energetic Particles (SEPs) are accelerated during solar eruptions up to relativistic energies, and propagate in the interplanetary medium to be observed by in situ instruments near Earth. The acceleration mechanisms of the SEPs, and their connection to other solar eruption manifestations, remain an unsolved question. To find an answer to this question, we must be able to interpret the in-situ observations of the Solar Energetic Particles, typically by spacecraft at 1 AU.

In the interplanetary space, the SEP propagation is guided by the interplanetary magnetic field, shaped as a Parker spiral which is superposed by turbulent fluctuations. The particles scatter off the turbulent fluctuations, which impedes their propagation along the field lines. On the other hand, the turbulence causes the field lines to meander, and the particles following the meandering fields propagate across the mean field line direction. The effect of the turbulence on the particles is typically modelled as diffusion, as described by the Fokker-Planck equation [1, 2], with field-aligned propagation modelled as pitch-angle diffusion [2], and the cross-field propagation as diffusion due to meandering of the field-lines [3]. Observations of galactic cosmic rays [4,5] and full-orbit particle simulations [6] support this description, and suggest for the ratio of the cross-field and field-aligned diffusion coefficients, $\kappa_{\perp} / \kappa_{\|} \sim 0.001-0.01$ for typical turbulence in the interplanetary space near Earth $[7,8,9]$.

However, recent multi-spacecraft observations suggest fast access of SEPs to wide range of heliographic longitudes, across the mean Parker spiral field, up to $180^{\circ}$ from the eruption location $[10,11]$. To reproduce these observations using the diffusion approach, much larger diffusion coefficient ratios, of order $\kappa_{\perp} / \kappa_{\|} \sim 0.1-1$ are needed [12, 13, 10, 14].

Recently, Laitinen et al [15] showed, using cartesian geometry, that the diffusion approach cannot reproduce the early evolution of SEP cross-field propagation. Instead, the SEPs propagate along the meandering field-lines deterministically until they decouple sufficiently to relax to diffusive behaviour. They introduced a model of SEP propagation, where the particles propagate and diffuse from turbulently meandering field-lines instead of the large-scale fields, and showed this model, FP+FLRW, to better describe the propagation of full-orbit simulated particles.

Following this work, Laitinen et al presented a FP+FLRW model for SEP propagation in Parker Spiral geometry, and showed how it can provide the observed rapid access of SEPs to wide range of longitudes [9]. In this report, we extend their work and show first results of the effect of interplanetary turbulence strength on the SEP event evolution.

\section{Models}

The traditional approach, the FP model, is based on solving for the distribution function $f$ using the Fokker-Planck equation [16, 17, 18]

$$
\begin{aligned}
& \frac{\partial f}{\partial t}+\left(\mu v \mathbf{b}+\mathbf{V}_{s w}\right) \cdot \nabla f+\frac{v}{2 L}\left(1-\mu^{2}\right) \frac{\partial f}{\partial \mu}+\left[\frac{\mu\left(1-\mu^{2}\right)}{2}\left(\nabla \cdot \mathbf{V}_{s w}-3 \mathbf{b b}: \nabla \mathbf{V}_{s w}\right)\right] \frac{\partial f}{\partial \mu} \\
= & \frac{\partial}{\partial \mu}\left(D_{\mu \mu} \frac{\partial f}{\partial \mu}\right)+\nabla \cdot \hat{\kappa} \nabla f,
\end{aligned}
$$

with $v$ and $\mu$ the particle's velocity and pitch-angle cosine, $\mathbf{V}_{s w}$ and $\mathbf{b}$ the solar wind velocity and a unit vector along the local mean magnetic field, respectively, and $L=-B /(\partial B / \partial s)$ the focusing 
length of the particles, with $s$ the arc-length along the field-line and $B$ the magnetic field. The pitchangle diffusion coefficient $D_{\mu \mu}$ is given by the quasi-linear theory (QLT) [2], and the cross-field diffusion coefficient $\kappa_{\perp}$, embedded in $\hat{\kappa}$, by the non-linear guiding centre theory (NLGC) [3].

The Eq. (2.1) is solved using stochastic differential equations (SDEs) [19], based on the code described in [20]. The particles are propagated in a Parker spiral field, with its magnitude $B$ given as

$$
B(r)=B_{0}\left(\frac{r_{0}}{r}\right)^{2} \sqrt{\frac{r^{2}+a^{2}}{r_{0}^{2}+a^{2}}},
$$

where $B_{0}=5 \mathrm{nT}$ is the magnetic field at heliocentric distance $r_{0}=1 \mathrm{AU}$, and $a=V_{s w} /\left(\Omega_{\odot} \sin \theta\right)$, where $V_{s w}=400 \mathrm{~km} / \mathrm{s}, \Omega_{\odot}=2.8631 \cdot 10^{-6} \mathrm{rad} / \mathrm{s}$ is the solar rotation rate and $\theta$ the co-latitude.

In the FP+FLRW model, the particles are propagated along field lines that random-walk across the Parker Spiral field. The meandering is described as field-line diffusion, and solved using SDEs, with the displacement $d r_{\perp}$ across the Parker field direction is solved with

$$
d r_{\perp}=\sqrt{2 D_{F L} d r_{\|}} W_{\perp}
$$

where $d r_{\|}$is the advance along the field, and $W_{\perp}$ a Gaussian random number with zero mean and unit variance. The field-line diffusion coefficient $D_{F L}$ is calculated as given in [21].

In addition to propagating along the meandering field-lines, the particles propagate diffusively across the meandering field and diffuse in pitch angle as in the FP model, with the QLT and NLGC diffusion coefficients $D_{\mu \mu}$ and $\kappa_{\perp}$. Such a model has been found to reproduce the early propagation of full-orbit simulated charged particles in turbulent magnetic fields [15].

The parameters for the FP and FP+FLRW models are calculated using a heliospheric turbulence model that consists of slab and 2D components [22],

$$
S(\mathbf{k}) \equiv S\left(\mathbf{k}, r_{0}\right)=S_{\perp}\left(k_{\perp}\right) \delta\left(k_{\|}\right)+S_{\|}\left(k_{\|}\right) \delta\left(\mathbf{k}_{\perp}\right),
$$

where $k_{\|}$and $k_{\perp}=\left|\mathbf{k}_{\perp}\right|$ are magnitudes of wave number, and the spectra $S_{\|}\left(k_{\|}\right)$and $S_{\perp}\left(k_{\perp}\right)$ are broken power laws with Kolmogorov spectra above breakpoint scale $L=0.007 \mathrm{AU}$, and constant and $\propto 1 / k_{\perp}$, respectively at larger scales up to the largest scale $L_{0}(r)=r$. The spectra are normalised by using the parallel scattering mean free path at $1 \mathrm{AU}, \lambda_{\|, 1 \mathrm{AU}} \equiv \lambda_{\|}(r=1 \mathrm{AU})$ as a parameter. In addition, we use the energy ratio $20 \%: 80 \%$ between slab and 2D components [8].

The turbulence spectra are evolved in space according to the WKB approximation [23, 24], with wave refraction, changes in the wave geometry and the modulus of $k$ neglected for simplicity. Using a constant radial solar wind velocity $V_{s w, r 0}$ and electron density $n_{e}(r)=n_{e 0} r_{0}^{2} / r^{2}$, we find

$$
S_{\|, \perp}\left(k_{\|, \perp}, r\right)=S_{\|, \perp}\left(k_{\|, \perp}, r_{0}\right)\left(\frac{r_{0}}{r}\right)^{3}\left(\frac{V_{s w, r 0}+v_{A 0}}{V_{s w, r 0}+\frac{r_{0}}{r} v_{A 0}}\right)^{2}
$$

where subscript 0 denotes the values at reference distance $r_{0}$, and $v_{A}$ is the Alfvén velocity. We use $V_{s w, r 0}=400 \mathrm{~km} / \mathrm{s}$ and $v_{a, r 0}=30 \mathrm{~km} / \mathrm{s}$ to represent the values at $r_{0}=1$ AU. Further details of the models are given in [9].

The ratio of the FP diffusion coefficients, $\kappa_{\perp} / \kappa_{\|}$, is shown in the left panel of Figure 1 for three different cases of interplanetary turbulence, as characterised by $\lambda_{\|, 1 \mathrm{AU}}=0.10 .3$ and $1 \mathrm{AU}$ 

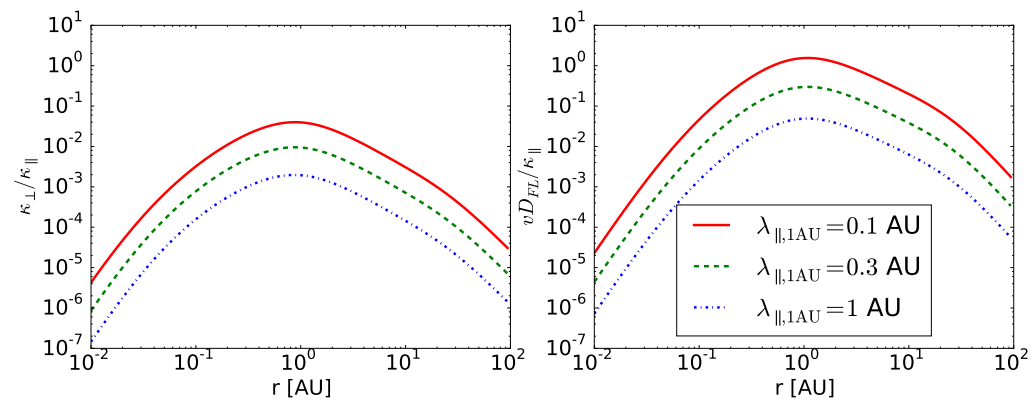

Figure 1: FP diffusion coefficient ratio (left panel) and the ratio of the $D_{F L}$, scaled with velocity, to $\kappa_{\|}$, for $10 \mathrm{MeV}$ SEP protons and different turbulence strengths parametrised by $\lambda_{\|, 1 \mathrm{AU}}$.
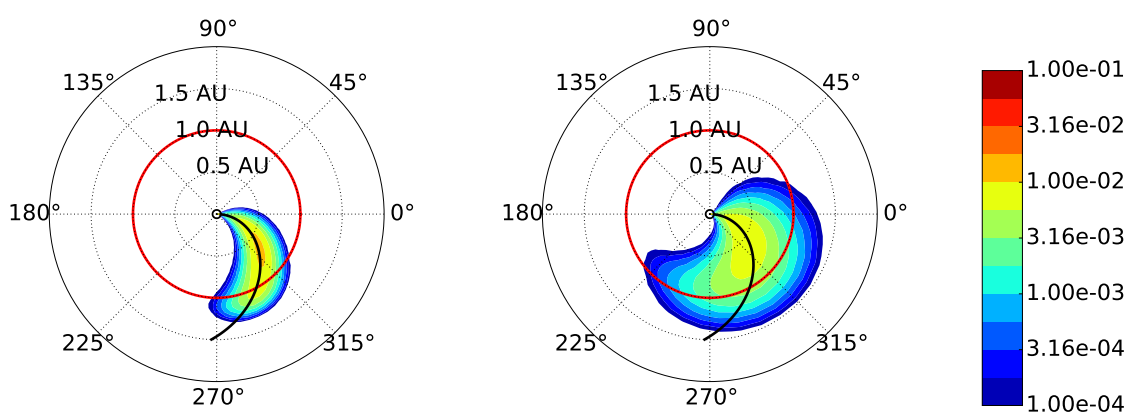

Figure 2: $10 \mathrm{MeV}$ SEP intensity, in arbitrary units, in ecliptic plane 2 hours after impulsive injection at $(r, \phi, \theta)=\left(1 \mathrm{r}_{\odot}, 0, \pi / 2\right)$, for the FP model (left panel) and the FP+FLRW model (right panel), respectively, with $\lambda_{\|, 1 \mathrm{AU}}=0.3 \mathrm{AU}$. The red curve depicts $1 \mathrm{AU}$ radial distance, and the thick black spiral curve the Parker field connected to the injection location.

for a $10 \mathrm{MeV}$ proton [25]. As can be seen, a decreasing parallel mean free path, due to stronger turbulence, results in larger cross-field diffusion coefficient, and as a result the ratio $\kappa_{\perp} / \kappa_{\|}$varies between 0.002 and 0.03 at $1 \mathrm{AU}$.

To depict the efficiency of the field line meandering in spreading the SEPs, we also show the cross-field diffusion coefficient for particles that beam along the meandering field line, giving the effective cross-field spreading in time as $\kappa_{\perp, F L R W}=v D_{F L}$. As can be seen in Figure 1, right panel, the diffusion coefficient ratio for such a cross-field diffusion coefficient exceeds the FP ratio by 1-2 orders of magnitude. This is in line with the findings of [15].

\section{Results and Discussion}

To compare the traditional FP model and the new FP+FLRW model we show in Fig. 2 the longitudinal extent of an SEP event at the equator 2 hours after $10 \mathrm{MeV}$ protons are released impulsively from a point source at the solar surface at zero latitude. For the case presented, the turbulence is parametrised with $\lambda_{\|, 1 \mathrm{AU}}=0.3 \mathrm{AU}$. As can be seen, two hours after the injection the difference between the FP model (left panel) and the FP+FLRW model (right panel) is large: in the FP model, the particles have spread diffusively only to a narrow cone of $60^{\circ}$ at $1 \mathrm{AU}$, whereas 

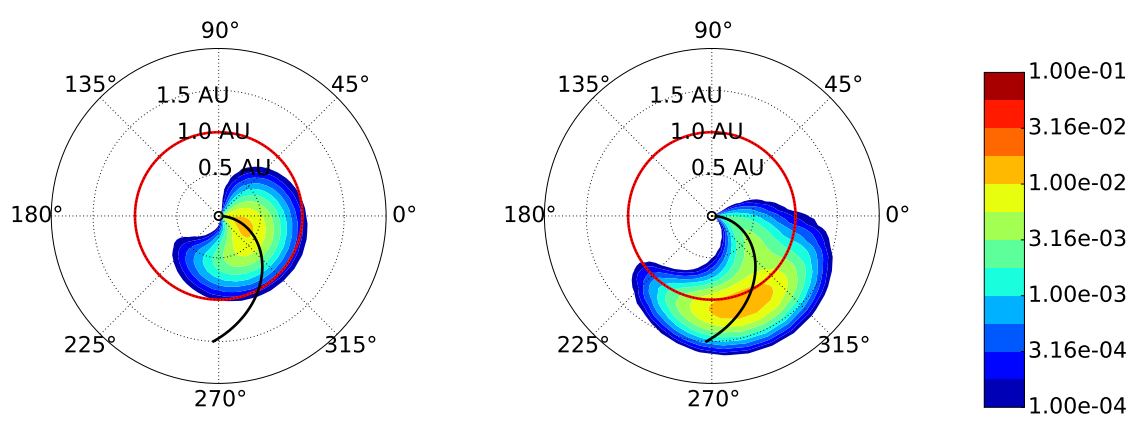

Figure 3: $10 \mathrm{MeV}$ SEP intensity, as in Fig. 2, with left panel for FP+FLRW with $\lambda_{\|, 1 \mathrm{AU}}=0.1 \mathrm{AU}$, and right panel for FP+FLRW with $\lambda_{\|, 1 \mathrm{AU}}=1 \mathrm{AU}$.

the meandering of the field-lines across the mean Parker field $160^{\circ}$ for similar intensity range. This case was analysed in further detail in [9], who showed that the FP+FLRW could explain not only the wide longitudinal extent of the observed SEP events, but also the observed fast access of SEPs to a wide range of longitudes. The extent of an SEP event simulated using the FP+FLRW method, as described by the longitudinal distribution of peak intensities, was fitted with a Gaussian with $\sigma=33^{\circ}$, consistent with the observed range of $\sigma=30^{\circ}-50^{\circ}[26,27,28,29,11]$. The FP model resulted in a considerably narrower event, with $\sigma=10^{\circ}$.

In this report, we show preliminary results of the effect of the level of turbulence to the SEP event evolution and extent. We keep the spectral shape and its radial evolution, as well as the energy ratio between the slab and $2 \mathrm{D}$ component as in [9]. However, we vary the turbulence amplitudes by varying $\lambda_{\|, 1 \mathrm{AU}}$, which is used to normalise the turbulence amplitudes, by setting it to values representing the observed strong and weak scattering conditions with $\lambda_{\|, 1 \mathrm{AU}}=0.1 \mathrm{AU}$ and $\lambda_{\|, 1 \mathrm{AU}}=1 \mathrm{AU}$, respectively [25]. The extent of $10 \mathrm{MeV}$ protons 2 hours after injection for these two cases is shown in Fig. 3.

As expected, the radial extent of the event depends strongly on the parallel mean free path, with the first particles having propagated to much larger distance in the case of $\lambda_{\|, 1 \mathrm{AU}}=1 \mathrm{AU}$ (right panel), compared to the stronger scattering presented in the left panel. As a consequence, after 2 hours, the protons have spread to a wider longitudinal area at $1 \mathrm{AU}$ distance from the Sun for the $\lambda_{\|, 1 \mathrm{AU}}=1$ AU case, as compared to the $\lambda_{\|, 1 \mathrm{AU}}=0.1 \mathrm{AU}$ case .

At later times, however, the $\lambda_{\|, 1 \mathrm{AU}}=0.1 \mathrm{AU}$ case results in a wider event than the $\lambda_{\|, 1 \mathrm{AU}}=1 \mathrm{AU}$ case. Our preliminary results suggest that the peak longitudinal width is considerably larger for the $\lambda_{\|, 1 \mathrm{AU}}=0.1 \mathrm{AU}$ case, as compared with the $\lambda_{\|, 1 \mathrm{AU}}=0.3 \mathrm{AU}$ case, but still within the observational range, whereas for $\lambda_{\|, 1 \mathrm{AU}}=1 \mathrm{AU}$ it remains below the $\sigma=30^{\circ}-50^{\circ}$ range. Thus, our results suggest that the observed widths of the SEP events can be explained by the varying solar wind turbulence environment.

\section{Conclusions}

As shown by recent analysis of multi-spacecraft -observed SEP events [10,14], the fast access of SEPS to a wide range of longitudes cannot be explained using the current framework of SEP 
transport modelling and our knowledge of the solar wind turbulence [7, 3]. We have presented results of the recently introduced model that incorporates field-line meandering, necessary to describe the initial cross-field spreading of SEPs [15], and showed that it is capable of describing these events (see also [9]). Our preliminary results show that the different levels of interplanetary turbulence can explain the variations observed in the extent of SEP events [26, 27, 28, 29, 11].

Acknowledgements. TL and SD acknowledge support from the UK Science and Technology Facilities Council (STFC) (grants ST/J001341/1 and ST/M00760X/1), MM from the European Commission FP7 Project COMESEP (263252), and FE from NASA grant NNX14AG03G. The contribution of AK benefitted from financial support through project He 3279/15-1, funded by the Deutsche Forschungsgmeinschaft (DFG). FE and SD acknowledge support from the International Space Science Institute as part of international team 297. We thank Horst Fichtner for helpful discussions. Access to the University of Central Lancashire's High Performance Computing Facility is gratefully acknowledged.

\section{References}

[1] E. N. Parker, The passage of energetic charged particles through interplanetary space, Planet. Space Sci. 13 (Jan., 1965) 9-49.

[2] J. R. Jokipii, Cosmic-Ray Propagation. I. Charged Particles in a Random Magnetic Field, ApJ 146 (Nov., 1966) 480-487.

[3] W. H. Matthaeus, G. Qin, J. W. Bieber, and G. P. Zank, Nonlinear Collisionless Perpendicular Diffusion of Charged Particles, ApJL 590 (June, 2003) L53-L56.

[4] R. A. Burger, M. S. Potgieter, and B. Heber, Rigidity dependence of cosmic ray proton latitudinal gradients measured by the Ulysses spacecraft: Implications for the diffusion tensor, J. Geophys. Res. 105 (Dec., 2000) 27447-27456.

[5] M. S. Potgieter, E. E. Vos, M. Boezio, N. De Simone, V. Di Felice, and V. Formato, Modulation of Galactic Protons in the Heliosphere During the Unusual Solar Minimum of 2006 to 2009, Sol. Phys. 289 (Jan., 2014) 391-406, [arXiv: 1302 .1284].

[6] J. Giacalone and J. R. Jokipii, The Transport of Cosmic Rays across a Turbulent Magnetic Field, ApJ 520 (July, 1999) 204-214.

[7] B. Bavassano, M. Dobrowolny, F. Mariani, and N. F. Ness, Radial evolution of power spectra of interplanetary Alfvenic turbulence, J. Geophys. Res. 87 (May, 1982) 3617-3622.

[8] J. W. Bieber, W. Wanner, and W. H. Matthaeus, Dominant two-dimensional solar wind turbulence with implications for cosmic ray transport, J. Geophys. Res. 101 (Feb., 1996) 2511-2522.

[9] T. Laitinen, A. Kopp, F. Effenberger, S. Dalla, and M. Marsh, Solar energetic particle access to distant longitudes via turbulent field-line meandering, ApJL (2015) [arXiv: 1508.03164]. Submitted.

[10] N. Dresing, R. Gómez-Herrero, A. Klassen, B. Heber, Y. Kartavykh, and W. Dröge, The Large Longitudinal Spread of Solar Energetic Particles During the 17 January 2010 Solar Event, Sol. Phys. 281 (Nov., 2012) 281-300.

[11] I. G. Richardson, T. T. von Rosenvinge, H. V. Cane, E. R. Christian, C. M. S. Cohen, A. W. Labrador, R. A. Leske, R. A. Mewaldt, M. E. Wiedenbeck, and E. C. Stone, > $25 \mathrm{MeV}$ Proton Events Observed by the High Energy Telescopes on the STEREO A and B Spacecraft and/or at Earth During the First $\sim$ Seven Years of the STEREO Mission, Sol. Phys. 289 (Aug., 2014) 3059-3107. 
[12] J. R. Dwyer, G. M. Mason, J. E. Mazur, J. R. Jokipii, T. T. von Rosenvinge, and R. P. Lepping, Perpendicular Transport of Low-Energy Corotating Interaction Region-associated Nuclei, ApJL 490 (Nov., 1997) L115+.

[13] M. Zhang, J. R. Jokipii, and R. B. McKibben, Perpendicular Transport of Solar Energetic Particles in Heliospheric Magnetic Fields, ApJ 595 (Sept., 2003) 493-499.

[14] W. Dröge, Y. Y. Kartavykh, N. Dresing, B. Heber, and A. Klassen, Wide longitudinal distribution of interplanetary electrons following the 7 february 2010 solar event: Observations and transport modeling, J. Geophys. Res. (Space Phys.) 119 (2014), no. 8 6074-6094.

[15] T. Laitinen, S. Dalla, and M. S. Marsh, Energetic Particle Cross-field Propagation Early in a Solar Event, ApJL 773 (Aug., 2013) L29, [arXiv: 1307 . 6362].

[16] E. C. Roelof, Propagation of Solar Cosmic Rays in the Interplanetary Magnetic Field, in Lectures in High-Energy Astrophysics (H. Ögelman and J. R. Wayland, eds.), p. 111, 1969.

[17] J. Skilling, Cosmic Rays in the Galaxy: Convection or Diffusion?, ApJ 170 (Dec., 1971) 265.

[18] M. Zhang, G. Qin, and H. Rassoul, Propagation of Solar Energetic Particles in Three-Dimensional Interplanetary Magnetic Fields, ApJ 692 (Feb., 2009) 109-132.

[19] C. W. Gardiner, Stochastic Methods, vol. 13. Springer-Verlag Berlin Heidelberg, 4 ed., 2009.

[20] A. Kopp, I. Büsching, R. D. Strauss, and M. S. Potgieter, A stochastic differential equation code for multidimensional Fokker-Planck type problems, Computer Physics Communications 183 (Mar., 2012) 530-542.

[21] W. H. Matthaeus, P. C. Gray, D. H. Pontius, Jr., and J. W. Bieber, Spatial Structure and Field-Line Diffusion in Transverse Magnetic Turbulence, Phys. Rev. Lett. 75 (Sept., 1995) 2136-2139.

[22] P. C. Gray, D. H. Pontius, and W. H. Matthaeus, Scaling of field-line random walk in model solar wind fluctuations, Geophys. Res. Lett. 23 (1996) 965-968.

[23] A. K. Richter and D. J. Olbers, Wave-Trains in the Solar Wind. II: Comments on the Propagation of Alfvén Waves in the Quiet Interplanetary Medium, Astrophys. Space Sci. 26 (Jan., 1974) 95-105.

[24] C.-Y. Tu, Z.-Y. Pu, and F.-S. Wei, The power spectrum of interplanetary Alfvenic fluctuations Derivation of the governing equation and its solution, J. Geophys. Res. 89 (Nov., 1984) 9695-9702.

[25] I. D. Palmer, Transport coefficients of low-energy cosmic rays in interplanetary space, Reviews of Geophysics and Space Physics 20 (May, 1982) 335-351.

[26] D. Lario, M.-B. Kallenrode, R. B. Decker, E. C. Roelof, S. M. Krimigis, A. Aran, and B. Sanahuja, Radial and Longitudinal Dependence of Solar 4-13 MeV and 27-37 MeV Proton Peak Intensities and Fluences: Helios and IMP 8 Observations, ApJ 653 (Dec., 2006) 1531-1544.

[27] D. Lario, A. Aran, R. Gómez-Herrero, N. Dresing, B. Heber, G. C. Ho, R. B. Decker, and E. C. Roelof, Longitudinal and Radial Dependence of Solar Energetic Particle Peak Intensities: STEREO, ACE, SOHO, GOES, and MESSENGER Observations, ApJ 767 (Apr., 2013) 41.

[28] M. E. Wiedenbeck, G. M. Mason, C. M. S. Cohen, N. V. Nitta, R. Gómez-Herrero, and D. K. Haggerty, Observations of Solar Energetic Particles from ${ }^{3}$ He-rich Events over a Wide Range of Heliographic Longitude, ApJ 762 (Jan., 2013) 54.

[29] N. Dresing, R. Gómez-Herrero, B. Heber, A. Klassen, O. Malandraki, W. Dröge, and Y. Kartavykh, Statistical survey of widely spread out solar electron events observed with STEREO and ACE with special attention to anisotropies, A\&A 567 (July, 2014) A27. 